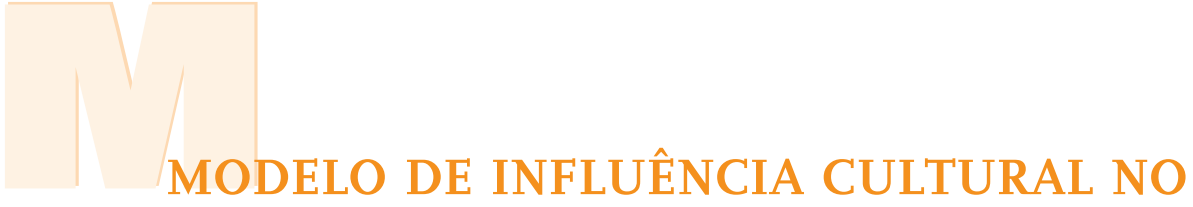 CONSUMO: UMA PROPOSTA BASEADA EM AXIOMAS SOCIAIS
}

\section{SOLANGE ALFINITO}

Doutora em Psicologia Organizacional, do Trabalho e das Organizações pelo Departamento de Psicologia Social e do Trabalho da Universidade de Brasília (UnB). Professora do Departamento de Administração e do Programa de Pós-Graduação em Administração da Universidade de Brasília (UnB).

Campus Universitário Darcy Ribeiro, Prédio FACE, Asa Norte, Brasília - DF - Brasil - CEP 70910-900

E-mail: solange.alfinito@gmail.com

\section{CLAUDIO V. TORRES}

Ph.D. em Industrial Organizational Psychology pela California School of Professional Psychology da University of California San Diego. Professor do Departamento de Psicologia Social e do Trabalho do Instituto de Psicologia da Universidade de Brasília (UnB). Campus Universitário Darcy Ribeiro, Asa Norte, Brasília - DF - Brasil - CEP 70910-900 E-mail: claudio.v.torres@gmail.com 


\section{RESUMO}

Escassos são os esforços no Brasil para investigar as relações entre o comportamento do consumidor e seus valores e suas crenças sociais (isto é, axiomas), embora existam evidências de que, também no país, os valores do consumidor explicam parte do processo de decisão de consumo, o que ajuda a sustentar a validade cultural do modelo das duas rotas (MDR). O objetivo do presente estudo foi avaliar a influência de aspectos culturais na decisão de compra do consumidor, indo além do MDR e propondo o modelo de influência cultural no consumo (Micc), que analisa a escolha do consumidor baseando-se não apenas nos valores, como também no construto de axiomas sociais proposto por Leung et al. (2002). Para testar o Micc, foi usado como objeto de estudo o mercado de educação superior brasileiro. Participaram 2.483 estudantes distribuídos em todo o país, com idade média de 22,6 anos ( $\mathrm{DP}=6,9$ ), sendo 59,2\% do sexo feminino. Eles responderam a uma medida de importância de atributos tangíveis, ao inventário de valores humanos de Schwartz (I994), à escala de axiomas sociais, à escala de julgamento e significado de serviços, além de dados demográficos. Após a verificação dos pressupostos e aplicação das análises fatoriais (PAF), foram conduzidas regressões logísticas binárias diretas e sequenciais usando somente valores, em seguida somente axiomas e depois, finalmente, valores e axiomas, como as variáveis-critério. Em termos gerais, os resultados apontam a estabilidade do Micc, que demonstrou representar uma alternativa superior ao MDR, após indicar melhoria preditiva de até $89,5 \%$. Discute-se que, enquanto valores são transituacionais e prescritivos, axiomas apresentam-se descritivos, além de cumprirem funções de atitudes relacionadas às descrições de objetos. Cognitivamente, a descrição do objeto social estaria mais disponível que a predição deste devido à maior proximidade dos atributos, possibilitando melhor explicação quanto ao comportamento de consumo. Os resultados têm implicações para um posicionamento mais eficaz no mercado, já que a sobrevivência nele depende também de aspectos subjetivos como aqueles envolvidos no Micc. 


\section{PALAVRAS-CHAVE}

Axiomas sociais; Valores humanos; Escolha do consumidor; Atributos; Modelo de influência cultural do consumo.

\section{INTRODUÇÃO}

A gênese de uma cultura envolve diversos aspectos, como crenças, valores e atitudes. São questões arraigadas em qualquer comportamento social, incluindo o de consumo. Apesar da existência de pesquisas que avaliam as relações entre o comportamento do consumidor e seus valores (ARNOULD; THOMPSON, 2005), Torres e Alfinito (2008) destacam que pouco tem sido estudado sobre o assunto no âmbito da cultura brasileira. Outra questão negligenciada se refere à investigação das decisões do consumidor perante as crenças sociais que tem. Trata-se de uma lacuna que precisa ser preenchida e que pode viabilizar a melhor compreensão sobre as expectativas dos consumidores a respeito de produtos e serviços, garantindo um posicionamento mais eficaz no mercado. Assim, este estudo avaliou a influência de aspectos culturais ligados a valores e crenças na decisão de compra do consumidor. Seu objetivo foi propor e testar um modelo de análise da escolha do consumidor, baseado em crenças sociais e valores humanos. Para a avaliação das crenças sociais, foi utilizado o construto de axiomas sociais de Leung et al. (2002).

Os valores humanos são definidos por Schwartz (I992) como metas motivacionais desejáveis para a vida. São princípios ou crenças intrinsecamente ligados à emoção, almejados como ideais para uma cultura. Os axiomas sociais, por sua vez, tentam descrever as crenças generalizadas existentes sobre um grupo social, exercendo um papel de organização de seu sistema de crenças e facilitando as relações interpessoais (LEUNG; BOND, 2004). Basicamente, são construtos complementares, já que os valores humanos preceituam o que se imagina como ideal para guiar as relações sociais, e os axiomas sociais descrevem como as pessoas percebem essas relações (ALFINITO, 2009).

Assim, o modelo proposto procurou conjugar os dois construtos para identificar a influência de seus diferentes olhares sobre o consumidor. O modelo resultante do estudo foi denominado modelo de influência cultural no consumo (Micc) e teve como base conceitual o modelo das duas rotas (MDR) desenvolvido por Allen (1997). Sugere-se aqui que, conquanto ancorado na abordagem do significado do produto, o Micc representa um avanço teórico-conceitual sobre o 
MDR ao incluir, em sua análise, os axiomas sociais, indo além dos valores humanos usados originalmente. A utilização de axiomas sociais no Micc surge como alternativa a modelos sociocognitivos, tradicionalmente empregados em comportamento do consumidor e marketing, que apresentam atitudes com variável central da relação atitude-comportamento (JACOBY; JOHAR; MORRIN, I998; SIMONSON et al., 200I).

Para a condução da pesquisa, o Micc foi aplicado no mercado de educação superior brasileiro, com a avaliação da escolha do estudante pela instituição de ensino superior (IES) em que se matriculou. A escolha da IES foi entendida como a escolha de um serviço (ROTHSCHILD; WHITE, I995) e os axiomas sociais foram testados como variáveis preditoras de escolha comparativamente aos valores humanos. Dessa forma, os objetivos específicos do estudo foram identificar se axiomas sociais (LEUNG et al., 2007) são melhores preditores de escolha do consumidor em relação a valores humanos (SCHWARTZ, I992; TAMAYO; SCHWARTZ, I993) e se a inclusão combinada das duas variáveis ao Micc melhoraria tal predição. Com vistas a atingir tais objetivos, apresenta-se a seguir um panorama sobre a área de comportamento do consumidor, seguido de uma breve revisão sobre os conceitos de valores humanos, axiomas sociais e outras variáveis que podem ser reunidas sobre a categoria de subjetividade do consumidor, as quais compõem a essência da proposta do Micc.

\section{COMPORTAMENTO DO CONSUMIDOR}

O consumo envolve todas as atividades mentais, emocionais e físicas que as pessoas desempenham quando selecionam, compram, usam e descartam produtos e serviços para satisfazer suas necessidades e seus desejos (STATT, I997). Trata-se de um processo complexo que começa muito antes de um produto ou serviço ser comprado e se estende até bem após o ato da compra, como esclarecem Flemming (I972) e Peter e Olson (I993) na teoria clássica de escolha do consumidor, behavioral decision theory (BDT - teoria de decisão comportamental).

Pela BDT, o primeiro estágio é aquele no qual o processo de decisão é ativado. As necessidades são percebidas e geram a intenção que reflete na escolha (KLEINDORFER; KUNREUTHER; SCHOEMAKER, I993). Em seguida o consumidor inicia sua busca por informações internas e externas sobre o produto ou serviço, processa a integração dessas informações, toma sua decisão e, finalmente, compra.

Durante a etapa que antecede a compra, a fase pré-compra, a atitude do consumidor em relação ao produto ou serviço é construída ou modificada sob a 
influência de diversos aspectos, como os grupos culturais de referência, a família e os esforços de marketing (JACOBY; HOYER; BRIEF, I992; SMITH; BOND, I999). Todavia, sua base de construção emerge dos valores e das crenças dos indivíduos, que podem elucidar aspectos importantes nesse primeiro estágio da BDT (EYAL; LIBERMAN; TROPE, 2009; MOOIJ, 2004).

Isso faz sentido ao se avaliar o silogismo proposto por Jones e Gerard (i967), no qual as crenças e os valores são usados como premissas. Avançando na proposta de Jones e Gerard (I967), outros autores, como Eagly e Chaiken (I998), sugerem que crenças seriam pensamentos não avaliativos referentes às características dos objetos do nosso mundo, que não necessariamente têm uma carga afetiva. Eagly e Chaiken (I998) ainda sugerem que os valores seriam avaliações quanto aos objetos do pensamento e, assim, pressupõem uma grande influência de questões afetivas. Pelo sistema de Jones e Gerard (I967), as atitudes são resultado da combinação lógica desses elementos cognitivos e valorativos. As atitudes compreenderiam, então, dois tipos de elemento: o cognitivo, que se refere às crenças, e o afetivo, que trata dos valores.

Em contrapartida, avaliar valores e crenças no contexto de consumo parece não fazer sentido, considerando que, como variáveis explicativas de comportamento de consumo, as psicossociais apresentam menor predição em detrimento de outras, como preço, qualidade, características do produto ou serviço etc. (ALLEN, I997, 2000; ALLEN; NG, I999; TORRES; ALFINITO; ALLEN, 2009). Isso pode incitar um possível engano. Se preço, qualidade, características do produto e outros aspectos mais tangíveis são as variáveis conhecidas e dominadas entre os concorrentes, elas não permitem que haja, na verdade, uma real diferenciação do produto, do serviço ou da marca no mercado e um consequente posicionamento adequado.

Nesse contexto, a sobrevivência no mercado depende do detalhe, do subjetivo, do pouco a mais que se pode entender sobre o consumidor. Aspectos ligados diretamente à demanda de mercado e não à oferta. Assim, construtos como o de valores humanos (SCHWARTZ, I992, I994; TAMAYO; SCHWARTZ, I993) têm sido aplicados em pesquisas empíricas em comportamento do consumidor (ALLEN, I997, 2000; TORRES; ALFINITO; PÉREZ-NEBRA, 2007; TORRES; ALFINITO; ALLEN, 2009) e evidenciado aspectos relevantes no processo de decisão do consumidor.

O modelo das duas rotas (MDR), proposto por Allen (I997, 2000), segue essa necessidade e procura detalhar claramente a relação dos valores com o processo de decisão de consumo. Ele tenta descrever a influência dos valores humanos (SCHWARTZ, I992) nas escolhas do consumidor. O MDR será mais bem explicitado adiante, após a avaliação comparativa entre os construtos de valores humanos e axiomas sociais. 


\section{VALORES HUMANOS E AXIOMAS SOCIAIS}

Os valores humanos e os axiomas sociais são construtos usados em pesquisas transculturais e se propõem, em essência, a avaliar as crenças das pessoas, a fim de prever comportamento social (LEUNG et al., 2002, 20II; SCHWARTZ; BILSKY, I987; SMITH; BOND; KAGITÇIBASI, 2006).

Prever o comportamento individual ou social não é tarefa simples, que poderá somente ser verificado por uma medida proximal. As crenças, os valores, os axiomas sociais e as atitudes formam, em conjunto, as bases que poderão dar a direção do comportamento dos indivíduos. Bond et al. (2004) investigaram a combinação de axiomas sociais e valores humanos na predição de comportamento social. Eles identificaram que a combinação desses dois construtos aumentou a predição dos comportamentos sociais examinados, como escolhas vocacionais, solução de conflitos e coping.

Mesmo apresentando similaridades teóricas e conceitos impregnados de afeto e crença, valores humanos e axiomas sociais são construtos que trazem propostas avaliativas distintas e complementares (ALFINITO, 2009; LEUNG et al., 2007). Os valores humanos se propõem a fazer uma avaliação mais prescritiva do indivíduo para a sua própria vida e apresentam um ponto de referência subjetivo. Eles são motivadores de comportamento e servem como orientadores no processo de julgamento de indivíduos e grupos (SCHWARTZ, I994). Os axiomas sociais são crenças compartilhadas sobre os ambientes social e físico e sobre o mundo espiritual, tidas como verdade em uma sociedade ou cultura (LEUNG et al., 2002). Eles se propõem a fazer uma avaliação mais descritiva quanto ao mundo social que o indivíduo percebe. Os axiomas permitem, assim, maior proximidade cognitiva em relação ao objeto social que os valores humanos.

\section{A SUBJETIVIDADE DO CONSUMIDOR}

É nos afetos humanos que se traduz o significado do que é consumido. Não é o produto, frio e distante, que importa. É o que ele significa. O significado resulta do processo de interpretação de estímulos externos. Assim, ele é compreendido como uma percepção subjetiva ou reação afetiva de uma pessoa diante de um objeto (RICHINS, I994). Essas percepções e reações se constituem e são influenciadas por relações interpessoais e sociais. Logo, significados individuais podem ser contrários aos sociais devido à subjetividade de cada indivíduo em seu processo de interpretação. 
Solomon (1983) argumenta que experiências subjetivas transmitidas pelo consumo de muitos produtos contribuem substancialmente para a estruturação da realidade social, do autoconceito e do comportamento do consumidor. Ou seja, os produtos são objetos simbólicos que representam papéis sociais dos outros e socializam o indivíduo em seus próprios papéis; e não simplesmente respostas a necessidades internas. São estímulos sociais que motivam os indivíduos a formar seu papel de autodefinição por meio de uma avaliação de autorreflexividade.

Nesse contexto, o MDR de Allen (1997) sugere que os valores humanos não influenciam a preferência pelo produto diretamente. Influenciam, na verdade, a importância dos atributos do produto, que, por sua vez, guiam a avaliação e compra dele. Outros modelos (TVERSKY; SATTATH; SLOVIC, I988), baseados no modelo de multiatributo de Fishbein (1963, 1967), indicam que os valores humanos se relacionariam à escolha do consumidor ao influenciarem os atributos do produto que os consumidores preferem. Essa seria uma avaliação calculada e racional dos atributos do produto que, por sua vez, determinaria a escolha do consumidor.

O MDR engloba essa avaliação por meio do julgamento racional do consumidor em relação aos aspectos tangíveis do produto, isto é, seu significado utilitário. Essa seria a primeira rota do modelo, mediada pelos atributos do produto. A segunda rota envolve uma avaliação afetiva e holística, o julgamento afetivo, no qual o significado simbólico do produto é o elemento central. Assim, o MDR representa uma maneira alternativa aos modelos de multiatributo para determinar como a escolha do produto é feita pelos consumidores e influenciada pelos valores endossados por eles, mediada por seus julgamentos e pelo significado que atribuem ao produto.

Mesmo sendo um modelo heterodoxo, sua base reside em valores que têm demonstrado baixo poder preditivo sobre o consumo. Ele já foi aplicado em pesquisas com diversos produtos e serviços, como automóveis, óculos de sol, itens alimentícios, pacotes de viagem, partidos políticos, entre outros (ALLEN, 200I; ALLEN; NG, I999; ALLEN; TORRES, 2006; NEPOMUCENO; PORTO; RODRIGUES, 2006; TORRES; ALFINITO; ALLEN, 2009; TORRES; ALFINITO; PÉREZ-NEBRA, 2007). Nesses estudos, os valores, nos construtos propostos por Schwartz (I994), Singelis et al. (I995) ou Rokeach (I973), apresentaram resultados satisfatórios na investigação intercultural, mas demonstraram considerável fragilidade no nível intracultural de análise de consumo. Isso abre uma lacuna para que haja a verificação da relação entre outros construtos e o consumo.

Este estudo apresenta uma possível alternativa para a avaliação do consumo, adicionando ao MDR os axiomas sociais propostos por Leung et al. (2002). 
O modelo-teste, denominado modelo de influência cultural no consumo (Micc) e apresentado na Figura I, é composto por duas versões: uma com os axiomas sociais como preditores de escolha e outra com a combinação de valores humanos e axiomas sociais como preditores. A investigação foi conduzida no mercado de educação superior no Brasil e buscou observar se os axiomas sociais poderiam ser a base das duas rotas do modelo em relação à instituição de ensino escolhida pelo estudante.

\section{FIGURA I}

PROPOSTA DO MODELO DE INFLUENCIA CULTURAL NO CONSUMO COM VALORES HUMANOS E AXIOMAS SOCIAIS COMO VARIÁVEIS PREDITORAS

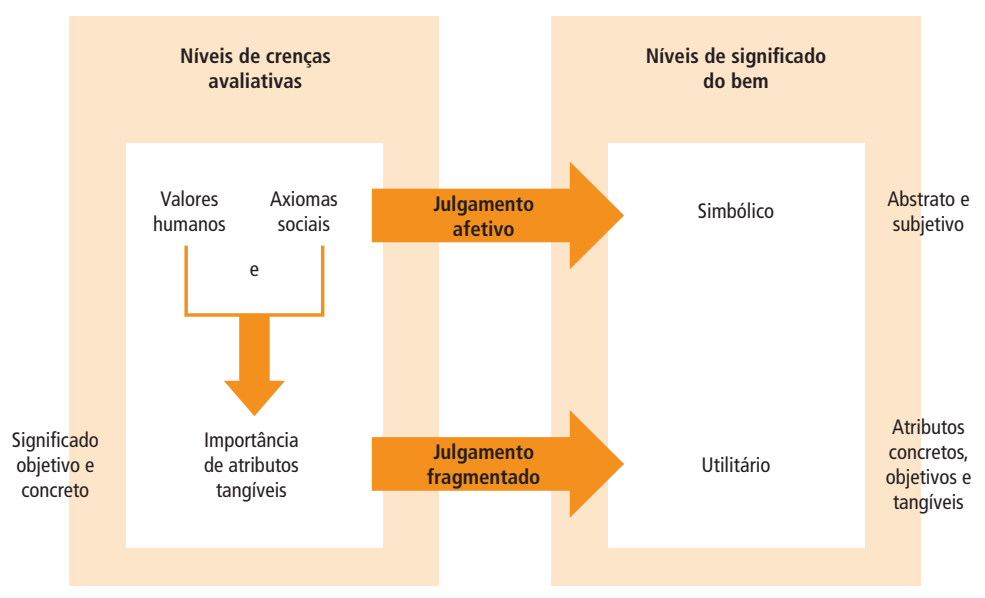

Fonte: Elaborada pelos autores com base em Allen (2000).

A escolha do estudante foi identificada de acordo com o tipo de instituição em que estava matriculado, respeitando os resultados da análise de cluster de Alfinito e Torres (2007), que indicaram que as IES brasileiras se agrupam em três categorias: grande porte, médio porte e pequeno porte. Além disso, vale observar que o Inep (20I0) também usa esse mesmo critério de porte para a classificação das IES brasileiras. Cada grupo possui características distintas, mas, em termos gerais, universidades e centros universitários compõem as de grande porte; faculdades integradas e centros tecnológicos, as de médio porte; faculdades isoladas, as de pequeno porte. A seguir, é apresentado o método com o detalhamento dos participantes, do instrumento e dos procedimentos de coleta de dados. 


\section{MÉTODO}

A pesquisa, quantitativa e exploratória, envolveu a condução de um survey via internet. A amostra foi não probabilística autogerada e, considerando apenas as respostas que foram usadas para o teste do modelo, envolveu 2.483 casos válidos. A média de idade dos entrevistados foi de 22,6 anos ( $D P=6,9$ ), sendo 59,2\% do sexo feminino e $50,2 \%$ da classe $B$, de acordo com o critério padrão de classificação econômica Brasil (ABEP, 2008, 20II). A maioria dos estudantes estava cursando o primeiro ano do ensino superior $(68,2 \%)$ e encontrava-se matriculada em IES privadas $(50,6 \%)$, das regiões Sul e Sudeste $(61,2 \%)$ do Brasil.

A divulgação do link de acesso à pesquisa foi feita em duas etapas de envio de e-mail, com intervalo de 20 dias entre elas. A base de dados dos e-mails foi o cadastro de cursos da educação superior de 2007 (INEP, 2008), contendo o contato dos gestores de todos os cursos presenciais no Brasil. Para preservar o princípio de anonimato dos respondentes, os coordenadores de curso foram convidados a encaminhar o endereço do sítio da pesquisa a, pelo menos, dez estudantes matriculados em curso superior sob sua responsabilidade.

O instrumento era autoaplicável e foi disponibilizado durante 40 dias consecutivos. Para minimizar o viés do respondente, todas as questões eram não obrigatórias. O instrumento foi apresentado em cinco blocos, contendo quatro escalas de medida tipo Likert e um bloco com perguntas abertas e fechadas para o levantamento dos dados sociodemográficos dos participantes. As escalas, um meio para que o objetivo da pesquisa fosse alcançado, foram testadas em relação às suas validades internas, e, para um melhor entendimento de cada uma, seus indicadores são descritos a seguir.

A primeira escala aplicada foi a dos atributos que serviram de variável mediadora da primeira rota do MDR. Seu objetivo foi mensurar aspectos relevantes na decisão em estudar na IES escolhida. A escala de atributos das IES (ALFINITO, 2009), com 35 itens, contou com escala de resposta tipo Likert, variando entre I - não importante - e 7 - muito importante. Por meio de análise fatorial exploratória com rotação oblíqua direta, foram retidos três fatores, identificados pelo teste scree de Cattel e confirmados pelo critério de análise paralela. O KMO foi de 0,92 e os fatores foram infraestrutura $(\mathrm{alfa}=0,92)$, imagem institucional (alfa $=0,8 \mathrm{I}$ ) e influência social (alfa $=0,74)$, com variância explicada de 44,05\%. Nove itens não compuseram nenhum fator por apresentarem cargas fatoriais e comunalidades baixas. O procedimento de corte dos itens, para todas as escalas, seguiu as recomendações de Churchill Jr. (I979), Lee e Hooley (2005) e Hair Jr. et al. (2005). 
Outra escala, utilizada para mensurar o construto de valores humanos, base do MDR, foi o inventário de valores humanos de Schwartz (IVS), organizado em dez dimensões motivacionais (PORTO, 2005; TAMAYO; SCHWARTZ, I993). Ela serviu para avaliar princípios orientadores na vida do indivíduo, contando com 6r itens em escala tipo Likert, variando entre I - não importante - e 7 muito importante.

Em seguida, foi apresentada a escala de julgamento e significado adaptada para serviços (ALLEN, 200I; NEPOMUCENO; TORRES, 2005), com 33 itens em escala tipo Likert, variando entre I - discordo totalmente - e 7 - concordo totalmente. O objetivo dessa escala foi levantar o tipo de julgamento feito pelo consumidor no processo de escolha, se racional ou afetivo, e o significado dado ao serviço, se utilitário ou simbólico. Para testar se essas quatro dimensões se aplicavam à amostra, foi conduzida uma análise fatorial com os mesmos procedimentos utilizados na escala de atributos. O KMO foi de o,88 e a estrutura de quatro fatores foi confirmada, com variância explicada de $16,95 \%$ e coeficientes de consistência interna variando entre o,64 e o,85. Dezesseis itens, com cargas fatoriais e comunalidades baixas, não apareceram em nenhum dos fatores.

A última escala aplicada foi a de axiomas sociais (LEUNG et al., 2002). Essa escala foi adaptada para o Brasil após processo de tradução reversa e validação semântica, resultando em 6I itens com escala tipo Likert de sete pontos (de I desacredito fortemente - a 7 - acredito fortemente) (ALFINITO; PÉREZ-NEBRA; TORRES, 2008). Sua finalidade é levantar as crenças generalizadas do ser social e seu ambiente, o que é feito em forma de assertivas que avaliam a relação entre duas entidades ou conceitos. Por meio de analise fatorial, também seguindo os critérios e procedimentos das fatoriais anteriores, foi alcançado um KMO de 0,90. Pela rotação oblíqua direta, foram retidos os cinco fatores previstos na teoria de Leung et al. (2002), com variância explicada de 32,60\%. As dimensões identificadas e seus respectivos coeficientes de consistência interna foram: ceticismo social $($ alfa $=0,65)$; complexidade social $($ alfa $=0,69)$; recompensa por empenho $(\mathrm{alfa}=0,73)$; religiosidade $($ alfa $=0,84)$; controle do destino $(\mathrm{alfa}=0,78)$. Destaca-se que esses índices foram superiores aos encontrados originalmente por Leung et al. (2002), que variaram entre 0,45 e $0,8 \mathrm{I}$.

Esclarecidos os aspectos relacionados à amostra, ao formato do instrumento, às características das variáveis envolvidas e aos procedimentos adotados para a coleta de dados, são apresentados, a seguir, os resultados encontrados na pesquisa. 


\section{RESULTADOS}

O modelo de influência cultural no consumo foi testado com base nos indicadores e na consistência interna das escalas de medida utilizadas. A variável critério, construída de acordo com a categorização de IES proposta por Alfinito e Torres (2007), consistiu na escolha por IES de grande porte em detrimento das demais.

Para o cálculo da influência das rotas direta e indireta, adotou-se o método padrão definido por Allen e Ng (I999). Esse método indica que os participantes sejam divididos em grupos não excludentes em termos de grau de julgamento e significado. Tal procedimento foi adotado no presente trabalho em relação a serviços. O primeiro grupo (denominado grupo A) compreendeu aqueles com alto grau de julgamento afetivo e significado simbólico ( $\mathrm{N}=\mathrm{I} .303$ ), caracterizando o grupo afetivo-simbólico. O grupo $\mathrm{B}$ refere-se àqueles com alto grau de julgamento fragmentado e significado utilitário ( $\mathrm{N}=$ I.374), constituindo o grupo fragmentado-utilitário. Para cada grupo, foram conduzidas regressões logísticas binárias diretas e sequenciais. Os pressupostos indicados para esse tipo de análise foram satisfeitos (LEECH; BARRET; MORGAN, 2005). O diagnóstico de colinearidade identificou a ausência de multicolinearidade, com VIF entre I,39 e 3,I9, desde que obedecido o limite de inclusão de oito dimensões de valores humanos em cada regressão, como salientado por Schwartz (2008). Nesse caso, as dimensões universalismo e benevolência não fizeram parte das análises por não serem, de acordo com a teoria de valores humanos, tipos motivacionais adjacentes ligados à escolha de IES (SCHWARTZ, 2005).

Devido ao grande número de regressões executadas, são apresentados na Tabela I apenas os resultados sumarizados das análises do modelo final original e das duas versões do modelo proposto. Para as diversas regressões executadas, o resultado da estatística Wald de cada equação foi significativo ( $\mathrm{p}<\mathrm{o,oI}$ ) em todos os casos. A influência da rota indireta seguiu a indicação de cálculo de Allen e Ng (I999) e sua significância não tem como ser calculada. Nas análises dos coeficientes específicos, os valores humanos de tradição, conformidade, segurança e poder, assim como os axiomas sociais de complexidade social e recompensa por empenho e o atributo infraestrutura, apresentaram Wald significativo. 


\section{TABELA I}

RESULTADOS DOS RS QUADRADOS DE NAGELKERIE

DA APLICAÇÃO DO MDR E MICC

\begin{tabular}{|c|c|c|c|c|c|c|c|}
\hline \multicolumn{8}{|c|}{ REGRESSÃO LOGÍSTICA SEQUENCIAL } \\
\hline & & & BLOCO 1 & BLOCO 2 & & & \\
\hline MODELO & $\begin{array}{c}\text { VARIÁVEL } \\
\text { TESTE }\end{array}$ & GRUPO & $\begin{array}{c}\text { IMPORTÂNCIA } \\
\text { DOS ATRIBUTOS } \\
\text { TANGÍVEIS }\end{array}$ & $\begin{array}{l}\text { FORÇA DA } \\
\text { VARIÁVEL } \\
\text { TESTE }\end{array}$ & $\begin{array}{c}\text { MUDANÇA } \\
N^{2} R^{2}\end{array}$ & $\begin{array}{l}\text { INFLUÊNCIA } \\
\text { DA ROTA } \\
\text { DIRETA }\end{array}$ & $\begin{array}{l}\text { INFLUÊNCIA } \\
\text { DA ROTA } \\
\text { INDIRETA }\end{array}$ \\
\hline \multirow{2}{*}{ MDR } & \multirow{2}{*}{ VH } & A & 0,065 & 0,093 & 0,028 & 0,046 & 0,018 \\
\hline & & B & 0,096 & 0,121 & 0,025 & 0,044 & 0,019 \\
\hline \multirow{2}{*}{$\begin{array}{c}\text { Micc } \\
\text { versão } 1\end{array}$} & \multirow{2}{*}{$A X$} & A & 0,065 & 0,089 & 0,024 & 0,050 & 0,026 \\
\hline & & B & 0,096 & 0,128 & 0,032 & 0,063 & 0,031 \\
\hline \multirow{2}{*}{$\begin{array}{c}\text { Micc } \\
\text { versão } 2\end{array}$} & \multirow{2}{*}{ VH e AX } & A & 0,065 & 0,107 & 0,042 & 0,072 & 0,030 \\
\hline & & B & 0,096 & 0,142 & 0,046 & 0,082 & 0,036 \\
\hline
\end{tabular}

Nota: $\mathrm{MDR}=$ modelo das duas rotas; Micc = modelo de influência cultural no consumo (versão $\mathrm{I}=$ com axiomas sociais; versão $2=\mathrm{com}$ valores humanos e axiomas sociais); $\mathrm{VH}=$ valores humanos; $\mathrm{AX}=$ axiomas sociais; grupo $\mathrm{A}=$ indivíduos com alto grau de julgamento afetivo e significado simbólico; grupo $\mathrm{B}=$ indivíduos com alto grau de julgamento racional e significado utilitário.

Fonte: Elaborada pelos autores.

Foi observado, no entanto, que, ao contrário do que sugerem Allen e Ng (I999), a separação da amostra em apenas dois grupos polarizados, cada um com alto grau de um tipo de julgamento e significado (afetivo-simbólico e fragmentado-utilitário), pode não refletir a realidade do comportamento de consumo para as rotas direta e indireta. Isso acontece porque os grupos obtidos, por serem não excludentes, têm, em sua composição, respondentes com altos escores nas díades afetivo-simbólico e fragmentado-utilitário. Logo, identificou-se que os indivíduos estão divididos, em verdade, em quatro grupos: dois remanescentes dos grupos A e B, aqui subdivididos em grupo I (com altos escores apenas em afetivo-simbólico, ou seja, seguidores exclusivos da rota direta, com $\mathrm{N}=458$ ) e grupo 2 (aqueles com altos escores apenas em fragmentado-utilitário e que utilizam unicamente a rota indireta, com $\mathrm{N}=529$ ), e dois outros grupos com a peculiaridade de serem definidos pelo alto grau de julgamento e significado nas duas rotas (ou seja, tanto racional quanto afetiva, compondo o grupo 3 , 
com $\mathrm{N}=854$ ) e baixo grau de julgamento e significado também nas duas rotas (compondo o grupo 4 , com $\mathrm{N}=65 \mathrm{I}$ ). Os quatro grupos foram submetidos a teste qui-quadrado, separados entre indivíduos que escolheram IES de grande porte e aqueles que não o fizeram. $O$ teste revelou uma associação significativa entre eles (qui-quadrado = I9,05; p < 0,0I), mesmo que subdivididos entre a escolha ou não. Em seguida, para verificar a pertinência da suposição dos quatro grupos no modelo, foram conduzidos testes de Kruskal-Wallis, indicados para dados não paramétricos, comparando-os em relação a cada uma das variáveis independentes do estudo. Todos os resultados foram significativos, exceto para os valores humanos de realização e hedonismo e para os axiomas sociais de ceticismo social e religiosidade.

O teste dos modelos foi novamente conduzido, agora com cada um dos quatro grupos identificados, e, considerando as variáveis significativas no teste de Kruskal-Wallis, os resultados das equações foram significativos $\left(\mathrm{p}_{\text {Wald }}<0,0 \mathrm{I}\right) \mathrm{e}$ estão descritos na Tabela 2. Os coeficientes específicos que apresentaram Wald significativo na maioria das regressões foram aqueles ligados aos valores humanos de tradição, segurança, estimulação e autodeterminação; os axiomas sociais de complexidade social e recompensa por empenho; e os atributos infraestrutura e influência social.

TABELA 2

RESULTADOS DOS RS QUADRADOS DE NAGELKERIE DA APLICAÇÃO DO MDR E MICC PARA OS QUATRO GRUPOS DE CONSUMIDORES

\begin{tabular}{|c|c|c|c|c|c|c|c|}
\hline \multicolumn{8}{|c|}{ REGRESSÃO LOGÍSTICA SEQUENCIAL } \\
\hline & & & BLOCO 1 & BLOCO 2 & & & \\
\hline MODELO & $\begin{array}{c}\text { VARIÁVEL } \\
\text { TESTE }\end{array}$ & GRUPO & $\begin{array}{l}\text { IMPORTÂNCIA } \\
\text { DOS ATRIBUTOS } \\
\text { TANGÍVEIS }\end{array}$ & $\begin{array}{l}\text { FORÇA DA } \\
\text { VARIÁVEL } \\
\text { TESTE }\end{array}$ & $\begin{array}{c}\text { MUDANÇA } \\
N^{2} R^{2}\end{array}$ & $\begin{array}{l}\text { INFLUÊNCIA } \\
\text { DA ROTA } \\
\text { DIRETA }\end{array}$ & $\begin{array}{l}\text { INFLUÊNCIA } \\
\text { DA ROTA } \\
\text { INDIRETA }\end{array}$ \\
\hline \multirow{4}{*}{ MDR } & \multirow{4}{*}{$\mathrm{VH}$} & 1 & 0,052 & 0,078 & 0,026 & 0,041 & 0,015 \\
\hline & & 2 & 0,114 & 0,135 & 0,021 & 0,023 & 0,002 \\
\hline & & 3 & 0,076 & 0,116 & 0,040 & 0,056 & 0,016 \\
\hline & & 4 & 0,057 & 0,099 & 0,042 & 0,042 & 0,000 \\
\hline
\end{tabular}


TABELA 2 (CONCLUSÃo)

\section{RESULTADOS DOS RS QUADRADOS DE NAGELKERKE \\ DA APLICAÇÃO DO MDR E MICC PARA OS QUATRO \\ GRUPOS DE CONSUMIDORES}

\begin{tabular}{|c|c|c|c|c|c|c|c|}
\hline \multicolumn{8}{|c|}{ REGRESSÃO LOGÍSTICA SEQUENCIAL } \\
\hline & & & BLOCO 1 & BLOCO 2 & & & \\
\hline MODELO & $\begin{array}{c}\text { VARIÁVEL } \\
\text { TESTE }\end{array}$ & GRUPO & $\begin{array}{c}\text { IMPORTÂNCIA } \\
\text { DOS ATRIBUTOS } \\
\text { TANGÍVEIS }\end{array}$ & $\begin{array}{c}\text { FORÇA DA } \\
\text { VARIÁVEL } \\
\text { TESTE }\end{array}$ & $\begin{array}{c}\text { MUDANÇA } \\
N^{2} R^{2}\end{array}$ & $\begin{array}{l}\text { INFLUÊNCIA } \\
\text { DA ROTA } \\
\text { DIRETA }\end{array}$ & $\begin{array}{l}\text { INFLUÊNCIA } \\
\text { DA ROTA } \\
\text { INDIRETA }\end{array}$ \\
\hline \multirow{4}{*}{$\begin{array}{c}\text { Micc } \\
\text { versão } \\
1\end{array}$} & \multirow{4}{*}{$A X$} & 1 & 0,052 & 0,061 & 0,009 & 0,029 & 0,020 \\
\hline & & 2 & 0,114 & 0,127 & 0,013 & 0,027 & 0,014 \\
\hline & & 3 & 0,076 & 0,120 & 0,044 & 0,071 & 0,027 \\
\hline & & 4 & 0,057 & 0,062 & 0,005 & 0,012 & 0,007 \\
\hline \multirow{4}{*}{$\begin{array}{c}\text { Micc } \\
\text { versão } \\
2\end{array}$} & \multirow{4}{*}{$\mathrm{VH}$ e $\mathrm{AX}$} & 1 & 0,052 & 0,083 & 0,031 & 0,054 & 0,023 \\
\hline & & 2 & 0,114 & 0,142 & 0,028 & 0,040 & 0,012 \\
\hline & & 3 & 0,076 & 0,143 & 0,067 & 0,098 & 0,031 \\
\hline & & 4 & 0,057 & 0,101 & 0,044 & 0,044 & 0,000 \\
\hline
\end{tabular}

Nota: $\mathrm{MDR}=$ modelo das duas rotas; Micc = modelo de influência cultural no consumo (versão $\mathrm{I}=\mathrm{com}$ axiomas sociais; versão $2=$ com valores humanos e axiomas sociais); $\mathrm{VH}=$ valores humanos; $\mathrm{AX}=$ axiomas sociais; grupo $\mathrm{I}=$ indivíduos com alto grau de julgamento afetivo e significado simbólico; grupo 2 = indivíduos com alto grau de julgamento racional e significado utilitário; grupo 3 = indivíduos com alto grau de julgamento e significado nas duas rotas; grupo 4 = indivíduos com baixo grau de julgamento e significado nas duas rotas.

Fonte: Elaborada pelos autores.

Para as todas as regressões executadas e descritas na Tabela 2, o resultado da estatística Wald para cada equação foi significativo ( $\left.\mathrm{p}_{\text {Wald }}<0, \mathrm{OI}\right)$. A influência da rota indireta seguiu o mesmo procedimento anterior, resultando em significância que não pode ser calculada. 


\section{DISCUSSÃO}

O presente estudo objetivou investigar uma possível alternativa de análise do comportamento de consumo, com base nos axiomas sociais de Leung et al. (2002), considerando o significado do produto, conforme aborda o MDR de Allen (I997), que parte dos valores humanos como influenciador na decisão de compra. Foram elaboradas, para tanto, duas propostas avaliativas em substituição aos valores humanos. A primeira com o uso dos axiomas sociais e a segunda com o uso combinado dos valores humanos com os axiomas sociais.

Adotou-se o critério de análise do MDR indicado em Allen e Ng (I999), com a divisão da amostra em dois grupos. Tanto para o grupo com maior grau de julgamento afetivo e significado simbólico (grupo A) como para o grupo com maior grau de julgamento fragmentado e significado utilitário (grupo B), conforme a Tabela I, foi identificada melhor predição com as duas versões propostas para o Micc, em ambas as rotas. Na primeira versão (apenas com axiomas sociais), o destaque foi para a rota indireta, que apresentou incremento de $44,4 \%$ para o grupo A e 63,2\% para o grupo B. Já na segunda versão (com valores humanos e axiomas sociais), o ganho foi claro nas duas rotas, chegando a $89,5 \%$ de melhora preditiva no grupo A, também na rota indireta.

A maior influência da rota indireta sobre a escolha com a aplicação dos axiomas sociais corrobora a abordagem teórica de Leung et al. (2002) para esse construto. Enquanto os valores humanos são transituacionais e prescritivos, os axiomas sociais se apresentam descritivos em relação às crenças sociais, além de cumprirem funções de atitudes relacionadas às descrições de objetos. Cognitivamente, a descrição de um objeto social estaria mais disponível que a predição dele devido à maior proximidade da realidade dos atributos. Isso ocorre porque, enquanto a prescrição envolve algo que se espera idealmente, a descrição envolve aquilo que se percebe como real. Essas mesmas características de prescrição e transituacionalidade dos valores humanos, no entanto, são adequadas para a rota direta, já que independem dos atributos ligados ao objeto social. Isso pôde ser observado, principalmente para o grupo A, com alto grau de julgamento afetivo e significado simbólico, no qual o aumento preditivo oferecido pelos axiomas sociais foi de apenas $8,7 \%$. Dessa forma, uma primeira conclusão a ser traçada aqui é que, como alternativa do MDR, os axiomas sociais caberão melhor como variável critério quando a importância dos atributos for elencada e, sob um julgamento fragmentado, o significado utilitário do bem (produto ou serviço) for o focalizado. Quando, porém, o significado simbólico for o mais relevante, envolvendo um julgamento afetivo, então os valores humanos aparecerão como a variável de melhor predição. Todavia, há que se ressaltar que não podemos, de antemão, prever qual significado será o preferido pelo consumidor, tampouco 
qual será o tipo de julgamento realizado no momento da escolha de diferentes produtos, serviços ou mesmo marcas.

Assim, ao se observar o caráter de complementaridade dos dois construtos testados - valores humanos e axiomas sociais (ALFINITO, 2009; LEUNG et al., 2007) -, nota-se um aumento considerável sobre a escolha com a aplicação da versão 2 do Micc. Quando se verifica apenas a quantidade de variância explicada por essa versão, fica clara a sua superioridade em relação às demais. Juntos, axiomas sociais e valores humanos apresentam-se como as melhores e mais poderosas variáveis critério em uma versão alternativa do MDR, promovendo predições mais fortes tanto para a rota indireta (com os axiomas sociais) quanto para a rota direta (com os valores humanos). É importante ressaltar, no entanto, que essa versão também apresenta limitações de ordem prática, já que envolve duas extensas escalas de medida, provocando, assim, maior desgaste para os consumidores avaliados. Sugere-se aqui que a solução mais adequada, tanto do ponto de vista teórico-empírico quanto do ponto de vista prático, seja a aplicação do modelo com escalas reduzidas desses construtos. Com essa solução, seria possível obter o acréscimo na explicação das variâncias, que foi apresentado no presente estudo para essa versão do Micc, seguida de uma menor carga cognitiva para os respondentes, diminuindo, assim, a mortalidade de sujeitos. Vale lembrar que, para o caso dos valores humanos, já foi apresentada, na literatura, uma escala de medida composta por 2I itens, também já validada para o Brasil (TAMAYO; PORTO, 2009). Para os axiomas sociais, novos estudos precisam ser conduzidos nesse sentido.

Outra questão a se considerar é a separação dos consumidores em apenas dois grupos antagônicos, sugerida por Allen e Ng (I999) e seguida em outros estudos (ALLEN, 200I; ALLEN; BAINES, 2002; ALLEN; TORRES, 2006). Evidenciou-se, no presente estudo, que os consumidores podem, na verdade, apresentar características distribuídas em quatro vertentes: indivíduos que utilizam exclusivamente a rota direta (grupo I); indivíduos que utilizam exclusivamente a rota indireta (grupo 2); e outros dois que acumulam extremos - indivíduos com alto grau de julgamento e significado nas duas rotas (grupo 3) e indivíduos com baixo grau de julgamento e significado também nas duas rotas (grupo 4). Com base nos resultados apresentados, pode-se afirmar que eles são realmente quatro grupos distintos, porém associados e com frequências adequadas entre os que escolheram IES de grande porte e os que não o fizeram. Outra verificação foi feita com o teste de Kruskal-Wallis, que mostrou diferença significativa entre os grupos em praticamente todas as covariantes do Micc.

Os resultados da aplicação do MDR e do Micc para os quatro grupos identificados evidenciaram que, ao contrário do que se imaginava, os indivíduos dos grupos essencialmente afetivo-simbólico ou fragmentado-utilitário não apre- 
sentam influências mais fortes nas rotas direta e indireta, respectivamente. Isso pode ocorrer devido à sobreposição de sujeitos quando se utilizam somente os dois grupos sugeridos no MDR, permitindo um viés nos resultados do modelo, com inflação dos coeficientes de determinação.

A rota direta apresentou-se fortalecida para os indivíduos com alto grau de julgamento (fragmentado e afetivo) e significado (simbólico e utilitário). Um ganho que variou entre $12,7 \%$ e 42,0\% em relação ao uso de apenas dois grupos nos modelos, como mostrado na Tabela I. Ressalta-se ainda que, para esse grupo 3, foi atingida uma predição de quase $10 \%$, com o uso combinado de axiomas sociais e valores humanos. Um indicador bastante promissor se for considerado o histórico preditivo baixo de variáveis desse tipo, o que pode estimular um olhar mais cuidadoso na importância da análise de valores e crenças no contexto de consumo. Fator que corrobora os estudos de Mooij (2004) e Eyal, Liberman e Trope (2009), ao indicarem a importância de variáveis desse tipo no primeiro estágio do processo de compra.

Para o grupo 4 - indivíduos com baixo julgamento e significado -, os resultados ficaram bem abaixo dos identificados na aplicação com dois grupos. Além de esperado, esse é um bom indicador, que confirma a importância da avaliação do julgamento e do significado no consumo, como já evidenciavam Richins (I994), Solomon (I983) e Allen (2000). Assim, quando os indivíduos são segmentados nos quatro grupos propostos, pode haver maior clareza de seu processamento em relação ao produto ou ao serviço-alvo. Ou seja, ao utilizar os quatro grupos, o pesquisador só tem a ganhar, pois pode observar maior variância e, consequentemente, maior explicação do fenômeno de interesse.

$\mathrm{Na}$ presente pesquisa, o Micc foi testado com estudantes de IES em todo o Brasil, com maior concentração, na amostra, daqueles matriculados em instituições de grande porte. Talvez essa seja uma limitação, já que escolher uma IES para estudar compreende um alto envolvimento do consumidor, o que pode ter estimulado a ocorrência de indivíduos com maior grau de julgamento e significado nas duas rotas, o grupo 3. Mesmo assim, o objetivo da pesquisa de propor e testar um modelo de influência cultural no consumo foi anterior a esse achado e pôde ser alcançado.

Como agenda futura de pesquisa, a escala de preferência de julgamento e significado de serviços demonstrou-se promissora e pode ser melhorada, bem como a de axiomas sociais. Esta última pode ser utilizada em versão reduzida. Quanto à aplicação do Micc, sugerem-se também outros setores além do educacional, seja com produtos, seja com serviços. Maior relevância do modelo poderia ser alcançada com sua aplicação em outros segmentos e mercados consumidores. Primeiramente, para algum produto e, posteriormente, para outro tipo de serviço. 
É importante destacar que, com a recente estabilização e emergência econômica do Brasil, atreladas ao processo de globalização sem precedentes, muitas oportunidades surgiram, mas também trouxeram ameaças aos gestores de marketing, como destacam Gammoh, Koh e Okoroafo (20II). Com a maior concorrência, as organizações são impelidas a apresentar vantagens competitivas em relação aos concorrentes, já que precisam de um bom posicionamento no mercado. Nesse cenário, aquelas que conseguirem essa diferenciação sobreviverão mais facilmente que as demais. Obviamente que se posicionar só é possível quando existe a conexão do posicionamento desejado para a marca ou para o produto, com a percepção, os valores e os desejos ou anseios já existentes nas mentes do público-alvo. Assim, o Micc torna-se ferramenta importante para o mercado, uma vez que permite a avaliação de relações entre variáveis psicossociológicas e a escolha do consumidor, possibilitando decisões mais precisas e melhor planejamento empresarial.

Dentre as limitações da pesquisa, há de se notar que 31,8\% da amostra de estudantes não estava cursando o primeiro ano do ensino superior, o que pode causar vieses de resposta, como um aumento de leniência decorrente da dissonância cognitiva, conforme descrita por Festinger (1957). Além disso, esse fato também pode refletir outro tipo de diferença: a de que estaríamos, em realidade, tratando com indivíduos de faixas etárias diferentes. Embora essa limitação se aplique a menos de um terço da amostra pesquisada, ainda assim ela deve ser controlada em pesquisas futuras.

Outra limitação diz respeito à não inclusão de outras variáveis no estudo, como o preço ou a renda, regularmente utilizados em análises econométricas. Assim, a princípio, apesar de o modelo obtido ter indicado um baixo poder explicativo - comprometendo sua utilização como importante ferramenta do mercado, a despeito do que foi afirmado anteriormente -, é mister lembrar que, quando se trabalha exclusivamente com variáveis sociais, intangíveis e, em tese, mais abstratas, essa limitação se converte em uma vantagem para o modelo, pois há a demonstração da relevante explicação fornecida por essas variáveis apesar da não utilização de variáveis mais duras.

Também deve ser observado o fato de que se obteve uma divisão quase igualitária entre o tipo de IES (pública versus privada) onde o respondente estava matriculado. Isso pode ser entendido como uma limitação, porque, em última análise, a escolha pela IES pública pode ter-se dado em função da sua gratuidade. Embora essa divisão quase igualitária (49,4\% dos estudantes estavam matriculados em IES públicas) possa ser entendida como uma forma de controle de variáveis (já que, com uma divisão equilibrada, na verdade, anulamos o efeito da variável tipo da IES nas análises), sugere-se que seu efeito seja testado em outros estudos que tenham, em seu escopo, especificamente esse objetivo. 


\section{CONSUMER CULTURAL INFLUENCE MODEL: A PROPOSAL BASED ON SOCIAL AXIOMS}

\section{ABSTRACT}

Few are the efforts in Brazil directed towards the investigation of the relationship between consumers' behaviors, values, and social beliefs (i.e., axioms), although there is evidence that in the country, consumer values also explain part of the consumption decision process, enhancing the cultural validity of the Two Route Model (TRM). This study's objective was to evaluate the influence of cultural aspects in the buying decision, going beyond the TRM, and proposing the Consumer Cultural Influence Model (CCIM), which analyses the consumer choice based not only on values, but also on the social axioms construct, as proposed by Leung et al. (2002). To test the CCIM, the Brazilian higher education market was chose as study object. Participated in the study 2,483 students from the entire country, with an average age of 22.6 years old $(\mathrm{SD}=6.9)$ and $59.2 \%$ of them female. They completed an importance of tangible attributes measure, the Schwartz Values Scale (SCHWARTZ, I994), the Social Axioms Scale, the Services Meaning and Judgment Scale, and demographics questions. After checking whether the assumptions were met, and Factor Analyses procedures, binary logistic regressions, among direct and sequential, were accomplished using only values, only axioms, and finally, values and axioms as criteria variables. In general, results show the stability of the CCIM, its superiority towards the TRM, with an increase in prediction of up to $89.5 \%$. Whilst values are transituational and prescriptive, axioms are descriptive, accomplishing instrumental functions of attitudes related to objects description. Cognitively, the description of a social object is more available to the person than its prediction, due to the proximity of the attributes to the object, allowing a better explanation of the consumer behavior. Results have implications to a more effective marketing positioning, as the survival in the market also depends on subjective aspects, such as those included in the CCIM.

\section{KEYWORDS}

Social axioms; Human values; Consumer choice; Attributes; Consumer cultural influence model. 


\section{MODELO DE INFLUENCIA CULTURAL SOBRE EL CONSUMO: UNA PROPUESTA BASADA EN AXIOMAS SOCIALES}

\section{RESUMEN}

Hay indicios de que los valores explican parte del proceso de decisión de consumo en Brasil, y esto ayuda a sostener la validez cultural del modelo de las dos rutas (MDR). Todavía, son escasos los esfuerzos para investigar la relación entre el comportamiento del consumidor y sus valores sociales y creencias (esto es, los axiomas) en el país. El objetivo de esta investigación fue evaluar la influencia de los aspectos culturales en las decisiones de compra, más allá del MDR, con la propuesta del modelo de influencia cultural sobre el consumo (Micc), que examina la elección del consumidor, con base no sólo en los valores, sino también por los axiomas sociales propuestos por Leung et al. (2002). Para probar el Micc se ha utilizado como objeto de estudio el mercado brasileño de enseñanza superior. Los participantes, 2.483 alumnos distribuidos en todo Brasil, tenían edad media de 22,6 años ( $D E=6,9)$, siendo 59,2\% mujeres. Ellos contestaran una medida de importancia de los atributos tangibles, el cuestionario de valores de Schwartz (I994), la escala de axiomas sociales, la escala de juicio y significado de los servicios, y datos demográficos. Después de comprobar los presupuestos y la aplicación de las análisis factoriales (PAF), se realizaron análisis de regresión logística binaria directas y secuenciales utilizando sólo los valores, sólo los axiomas y, por último, los valores y axiomas, como las variables criterio. En general, los resultados indican la estabilidad del Micc, que demostró representar una alternativa superior a el MDR, después de indicar una mejor predicción de hasta el 89,5\%. Se argumenta que mientras los valores son transituacionales y prescriptivos, los axiomas se presentan como descriptivos, además de cumplir con las funciones de las actitudes relacionadas con las descripciones de los objetos. Cognitivamente, la descripción del objeto social estaría más disponible que su predicción debido a la mayor proximidad a los atributos, lo que permite una mejor explicación del comportamiento de consumo. Los resultados tienen implicaciones para un posicionamiento más efectivo en el mercado, ya que la supervivencia en el depende también de aspectos subjetivos como los que participan en el Micc.

\section{PALABRAS CLAVE}

Axiomas sociales; Valores humanos; Elección del consumidor; Atributos; Modelo de influencia cultural sobre el consumo. 


\section{REFERÊNCIAS}

ABEP. Critério padrão de classificação econômica Brasil. 2008. Disponível em: <http://www.abep. org/default.aspx? usaritem=arquivos\&iditem=23>. Acesso em: 20 mar. 2008.

ABEP. Critério padrão de classificação econômica Brasil. 20II. Disponível em: <http://www.abep. org/novo/Utils/FileGenerate.ashx?id=I97>. Acesso em: 7 jun. 20 II.

ALFINITO, S. A influência de valores humanos e axiomas sociais na escolha do consumidor: uma análise comparativa aplicada à educação superior. 2009. I32 f. Tese (Doutorado em Psicologia Social, do Trabalho e das Organizações)-Instituto de Psicologia, Universidade de Brasília, Brasília, 2009. ALFINITO, S.; PÉREZ-NEBRA, A. R.; TORRES, C. V. Escala de axiomas sociais para o Brasil: adaptação e análise de consistência interna com base no Inventário de Valores de Schwartz. In: SIMPÓSIO INTERNACIONAL DE VALORES HUMANOS E GESTÃO, 2., 2008, São Paulo. Anais... São Paulo: UPM, 2008.

ALFINITO, S.; TORRES, C. V. Higher education institutions in Brazil: a brand clustering proposal. In: LATIN AMERICAN CROSS-CULTURAL CONGRESS, 4., 2007, Cidade do México. Anais... Cidade do México: IACCP, 2007.

ALLEN, M. W. The direct and indirect influences of human values on consumer choices. 1997. $342 \mathrm{p}$. Tese (Doutorado em Psicologia)-Victoria University of Wellington, New Zealand, I997.

ALLEN, M. W. The attribute-mediation and product meaning approaches to the influences of human values on consumer choices. In: COLUMBUS, F. (Org.). Advances in psychology research. Huntington, NY: Nova Science Publishers, 2000. v. I, p. 33-76.

ALLEN, M. W. A practical method for uncovering the direct and indirect relationships between human values and consumer purchases. Journal of Consumer Marketing, v. I8, n. 2, p. IO2-I20, 200 I. ALLEN, M. W. Human values, utilitarian benefits and identification: the case of meat. European Journal of Social Psychology, v. 33, n. I, p. 37-56, 2002.

ALLEN, M. W.; BAINES, S. Manipulating the symbolic meaning of meat to encourage greater acceptance of fruits and vegetables and less proclivity for red and white meat. Appetite, v. 38, n. 2, p. II8-I30, 2002.

ALLEN, M. W.; NG, S. H. The direct and indirect influence of human values on product ownership. Journal of Economic Psychology, v. 20, n. I, p. 5-39, I999.

ALLEN, M. W.; TORRES, C. V. Food symbolism and consumer choice in Brazil. In: GONZALEZ, S.; LUNA, D. (Org.). Latin American advances in consumer research. Monterey, México: Association for Consumer Research, 2006. p. 32-45.

ARNOULD, E. J.; THOMPSON, C. J. Consumer culture theory (CCT): twenty years of research. Journal of Consumer Research, v. 3I, n. 4, p. 868-882, 2005.

BOND, M. H. LEUNG, K.; AU, A.; TONG, K.; CHEMONGES-NIELSON, Z. Combining social axioms with values in predicting social behaviours. European Journal of Personality, v. I8, p. I77I9I, 2004 .

CHURCHILL JR., G. A. A paradigm for developing better measures of marketing constructs. Journal of Marketing Research, v. I6, p. 64-73, I979.

EAGLY, A. H.; CHAIKEN, S. Attitude structure and function. In: GILBERT, D. T.; FISKE, S. T.; LINDZEY, G. (Ed.). The handbook of social psychology, 4. ed. Boston, MA: McGraw-Hill, I998. v. I, p. 269-322. 
EYAL, T.; LIBERMAN, N.; TROP, Y. Psychological distance and consumer behavior: a construal level theory perspective. In: WÄNKE, M. (Ed.). Social psychology of consumer behavior. New York: Psychology Press, 2009. p. 65-88.

FESTINGER, L. Theory of cognitive dissonance. California: Stanford Press, I957.

FISHBEIN, M. An investigation of the relationships between beliefs about the object and the attitude toward that object. Human Relations, v. I6, p. 233-240, I963.

FISHBEIN, M. Readings in attitude theory and measurement. New York: Wiley, I967.

FLEMMING, H. Consumer choice behavior: a cognitive theory. New York: The Free Press, I972.

GAMMOH, B. S.; KOH, A. C.; OKOROAFO, S. C. Consumer culture brand positioning strategies: an experimental investigation. Journal of Product \& Brand Management, v. 20, n. I, p. 48-57, 201 I. HAIR JR., J. F.; ANDERSON, R. E.; TATHAM, R. L.; BLACK, W. C. Análise multivariada de dados. Porto Alegre: Bookman, 2005 .

INEP. Cadastro de cursos da educação superior. Brasília: Inep, 2008. Disponível em: <http://www. educacaosuperior.inep.gov.br/inst.stm>. Acesso em: 26 mar. 2008.

INEP. Resumo técnico: censo da educação superior de 2009. Brasília: Inep, 20Io. Disponível em: $<$ http://download.inep.gov.br/download/superior/censo/2009/resumo_tecnico2009.pdf>. Acesso em: 5 abr. 2011.

JACOBY, J.; HOYER, W.; BRIEF, A. Consumer psychology. In: DUNNETTE, M. D.; HOUGH, L. M. (Org.). Handbook of industrial and organizational psychology. California: Consulting Psychologists Press, I992. v. 3, p. 377-44I.

JACOBY, J.; JOHAR, G. V.; MORRIN, M. Consumer behavior: a quadrennium. Annual Review of Psychology, v. 49, p. 319-344, I998.

JONES, E. E.; GERARD, H. B. Foundations of social psychology. New Jersey: J. Wiley, I967.

KLEINDORFER, P. R.; KUNREUTHER, H. C.; SCHOEMAKER, P. J. H. Decision sciences: an integrative perspective. Cambridge: Cambridge Press, I993.

LEE, N.; HOOLEY, G. The evolution of "classical mythology" within marketing measure development. European Journal of Marketing, v. 39, n. 3-4, p. 365-385, 2005.

LEECH, N. L.; BARRET, K. C.; MORGAN, G. A. SPSS for intermediate statistics: use and interpretation. New Jersey: Lawrence Erlbaum, 2005.

LEUNG, K.; BOND, M. H. Social axioms: a model of social beliefs in multi-cultural perspective. In: ZANNA, M. P. (Org.). Advances in experimental social psychology. San Diego, CA: Academic Press, 2004. v. 36, p. II9-I97.

LEUNG, K.; AU, A.; HUANG, X.; KURMAN, J.; NIIT, T.; NIIT, K. Social axioms: the search for universal dimensions of general beliefs about how the world functions. Journal of Cross-Cultural Psychology, v. 33, n. 3, p. 286-302, 2002.

LEUNG, K.; AU, A.; HUANG, X.; KURMAN, J.; NIIT, T.; NIIT, K. Social axioms and values: a cross-cultural examination. European Journal of Personality, v. 2I, p. 9I-III, 2007.

LEUNG, K.; LAM, B. C. P.; BOND, M. H.; CONWAY, L. G.; GORNICK, L. J.; AMPONSAH, B.; BOEHNKE, K. Developing and evaluating the social axioms survey in eleven countries: its relationship with the five-factor model of personality. Journal of Cross-Cultural Psychology, setembro, doi:Io.II77/0022022III4I636I, 20II.

MOOIJ, M. Consumer behavior and culture: consequences for global marketing and advertising. London: Sage, 2004 . 
NEPOMUCENO, M. V.; PORTO, R. B.; RODRIGUES, H. Avanços teóricos e metodológicos das pesquisas sobre julgamento e significado do produto no Brasil. Psicologia: organizações e trabalho, v. 6, n. I, p. Iо6-I37, 2006.

NEPOMUCENO, M. V.; TORRES, C. V. Validação da escala de julgamento e significado do produto. Estudos de Psicologia, Natal, v. IO, n. 3, p. 42I-430, 2005.

PETER, J. P.; OLSON, J. C. Consumer behavior and marketing strategy. Homewood: Richard D. Irwin, I993.

PORTO, J. B. Mensuração de valores no Brasil. In: TAMAYO, A.; PORTO, J. B. (Org.). Valores e comportamentos nas organizações. Petrópolis: Vozes, 2005. p. 97-II9.

RICHINS, M. L. Valuing things: the public and private meanings of possessions. Journal of Consumer Research, v. 2I, p. 504-52I, I994.

ROKEACH, M. The nature of human values. New York: Free Press, I973.

ROTHSCHILD, M.; WHITE, L. J. The analytics of the pricing of higher education and other services in which the customers are inputs. Journal of Political Economy, Chicago, v. I03, n. 3, p. 573586, June i995.

SCHWARTZ, S. H. Universals in the content and structure of values: theoretical advances and empirical tests in 20 countries. In: ZANNA, M. P. (Org.). Advances in experimental social psychology. Orlando, FL: Academic Press, I992. v. 25, p. I-65.

SCHWARTZ, S. H. Are there universal aspects in the structure and contents of human values? Journal of Social Issues, v. 50, n. 4, p. 19-45, I994.

SCHWARTZ, S. H. Valores humanos básicos: seu contexto e estrutura intercultural. In: TAMAYO, A.; PORTO, J. B. (Org.). Valores e comportamentos nas organizações. Petrópolis: Vozes, 2005. p. 2I-55. SCHWARTZ, S. H. Computing scores for io human values. Disponível em: <http://ess.nsd.uib.no/ files/2003/ESSiCodingHumanValueScale.doc>. Acesso em: 8 set. 2008.

SCHWARTZ, S. H.; BILSKY, W. Toward a universal psychological structure of human values. Journal of Personality and Social Psychology, v. 3, p. 550-562, I987.

SIMONSON, I.; CARMON, Z.; DHAR, R.; DROLET, A.; NOWLIS, S. M. Consumer research: in search of identity. Annual Review of Psychology, v. 52, p. 249-275, 200 I.

SINGELIS, T. M.; TRIANDIS, H. C.; BHAWUK, D. P. S.; GELFAND, M. J. Horizontal and vertical dimensions of individualism and collectivism: a theoretical and measurement refinement. CrossCultural Research, v. 29, n. 3, p. 240-275, 1995.

SMITH, P. B.; BOND, M. H. Social psychology across cultures. Boston: Allyn \& Bacon, I999.

SMITH, P. B.; BOND, M. H.; KAGITÇIBASI, Ç. Understanding social psychology across cultures: living and working in a changing world. London: Sage, 2006.

SOLOMON, M. R. The role of products as social stimuli: a symbolic interactionism perspective. Journal of Consumer Research, v. I0, n. 3, p. 319-329, I983.

STATT, D. A. Understanding the consumer: a psychological approach. London: MacMillan, I997.

TAMAYO, A.; SCHWARTZ, S. H. Estrutura motivacional dos valores humanos. Psicologia: teoria e pesquisa, v. 9, n. 2, p. 329-348, I993.

TORRES, C. V.; ALFINITO, S. Cultura e o comportamento do consumidor: os valores culturais são preditivos do consumo? In: TEIXEIRA, M. L. M. (Org.). Valores humanos \& gestão: novas perspectivas. São Paulo: Senac, 2008. p. II7-I4I. 
TORRES, C. V.; ALFINITO, S. ALLEN, M. W. Influência da cultura, dos valores humanos e do significado do produto na predição de consumo: síntese de dois estudos multiculturais na Austrália e no Brasil. Revista de Administração Mackenzie, v. Io, n. 3, p. I27-I52, 2009.

TORRES, C. V.; ALFINITO, S.; PÉREZ-NEBRA, A. R. The influence of human values on holiday destination choice in Australia and Brazil. Brazilian Administration Review, v. 4, n. 3, p. 63-76, 2007. TVERSKY, A.; SATTATH, S.; SLOVIC, P. Contingent weighting in judgment and choice. Psychological Review, v. 95, p. 37I-384, I998. 